Journal of Thermal Engineering, Vol. 5, No. 5, pp. 396-404, October, 2019

Yildiz Technical University Press, Istanbul, Turkey

\title{
THE INFLUENCE OF TEMPERATURE ON DISPERSION OF PARTICLES ON LIQUID SURFACES
}

\author{
S. K. Gurupatham ${ }^{1, *}$, V. Sooklal ${ }^{1}$, A. Hensen ${ }^{1}$, M. Conner ${ }^{1}$, M. Sayedzada1 ${ }^{1}$, D. Shah ${ }^{1}$
}

\begin{abstract}
It was shown recently that small particles and powders spontaneously disperse on liquid surfaces when they come in contact with the interface for the first time (Figure 2). This happens due to the combined effect of the capillary force, buoyant weight of the particle and the viscous drag that the particles experience when they encounter the liquid surface. The particles undergo oscillations normal to the interface before they come to the equilibrium position on the interface. These oscillations, in turn, induce a flow on the interface which disperses the particles radially outward. This phenomenon has a significant role in the pollination of sea plants such as "Ruppia" in which the formation of "pollen rafts" is the first step which results from the spontaneous dispersion of their pollens on the water surface. This work investigates, experimentally, the influence of temperature of the liquid on which this dispersion occurs. It was observed that the frequency of oscillations of the particles decreased with the increase in the temperature of the liquid. It is because the magnitude of capillary force that the particle experiences also decreased when the temperature of the liquid increased.
\end{abstract}

Keywords: Adsorption, Surface Tension, Particle Dispersion Fluid-Liquid Interface, Capillary Force, Viscous Drag

\section{INTRODUCTION}

In recent years, the understanding of adsorbed state of particles at fluid-liquid interfaces is gaining importance due to their numerous applications. Some of the areas which come under this are self-assembly of particles at fluidliquid interfaces, the stabilization of emulsions, the pollination in hydrophilous plants, the floatation of insect eggs, the dispersion of viruses and macromolecules, etc. [1-9].

When small particles and powders come in contact with a liquid surface they disperse violently, initially and then come back to form clusters [10]. The particles experience a pulling force towards their equilibrium position by the vertical component of capillary force when they touch the liquid surface by which the particles attain high velocity in the vertical direction (Figure 1(a) to (c)). This high velocity leads to overshooting the equilibrium position of the particles since the drag force is not enough for small particles to control the motion in the downward direction. When the particle passes the equilibrium height (which is the height between the particle center and the undeformed liquid surface) because of the overshooting, the direction of the capillary force reverses its direction of action which also reverses the direction of the motion of the particles in upward direction. This leads to the oscillation of the particles about the interface in the vertical direction which consequently induces a surface flow laterally which in turn causes the dispersion of these particles explosively on the surface in the radial direction. The mathematical expression for the initial velocity of the particles when it touches the interface was shown as: 


$$
V=\frac{-\frac{9}{4} \mu+\sqrt{\frac{81}{16} \mu^{2}+4 R \rho_{p}\left(\frac{3}{2} \gamma+2 R^{2}\left(\rho_{\mathrm{p}}-\rho_{\mathrm{c}}\right) g\right)}}{2 R \rho_{p}}
$$

where $\rho_{\mathrm{c}}$ is the effective fluid density which changes while the particle moves normal to the interface, $\rho_{\mathrm{p}}$ is the particle density, $\gamma$ is the interfacial tension between the upper and lower fluids, $\mathrm{R}$ is the radius of particle and $\mu$ is the viscosity of the lower fluid. The frequency of oscillation of the particle about the interface was also shown as:

$$
\omega=\frac{3 \sqrt{-9 R^{2} \mu^{2}+\frac{16}{3} R^{3} \rho_{\mathrm{p}}\left(2 \gamma+R^{2}\left(\rho_{\mathrm{p}}-\rho_{\mathrm{c}}\right) g\right)}}{16 \pi R^{3} \rho_{\mathrm{p}}}
$$

The initial explosive dispersion lasts for a short time usually one or two seconds depending on the liquid on which the dispersion occurs and later the clustering phase of particles start which is dominated by the attractive capillary forces [11-17], and [19]. Sometimes, the particles could remain dispersed if they are of very small size (very small micron and nano-sized particles) since their capillary forces are insignificant.

Also, it was shown that some small particles might experience lateral forces such as electrostatic, Brownian, etc., which might result in the cluster or some patterns [21], [18], [23], [24], and [17].

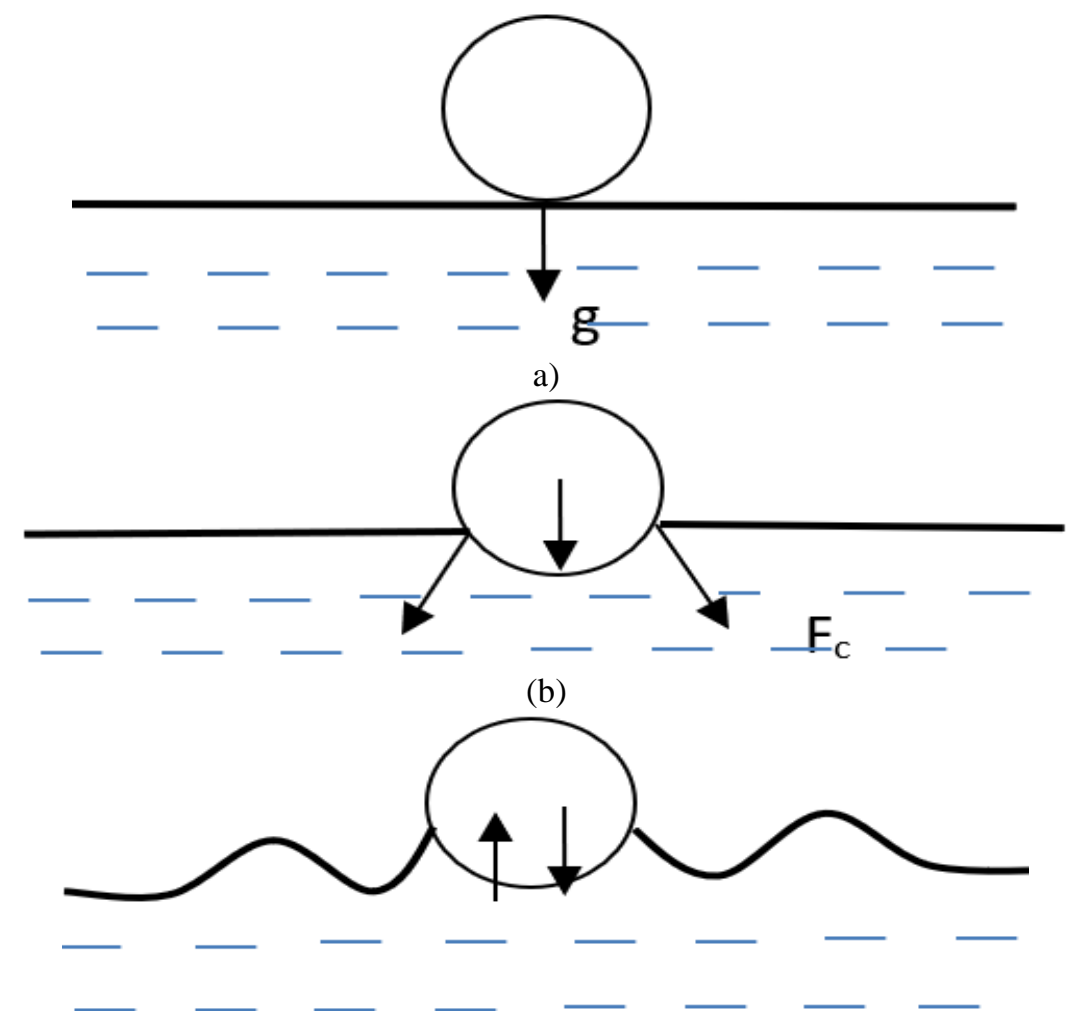

(c)

Figure 1. (a) The adsorption of a particle on the air-liquid interface, (b) The particle is pulled by the capillary force downward along with the gravity, (c) The spontaneous dispersion of turmeric powder of approximately 30 microns in size on water surface 


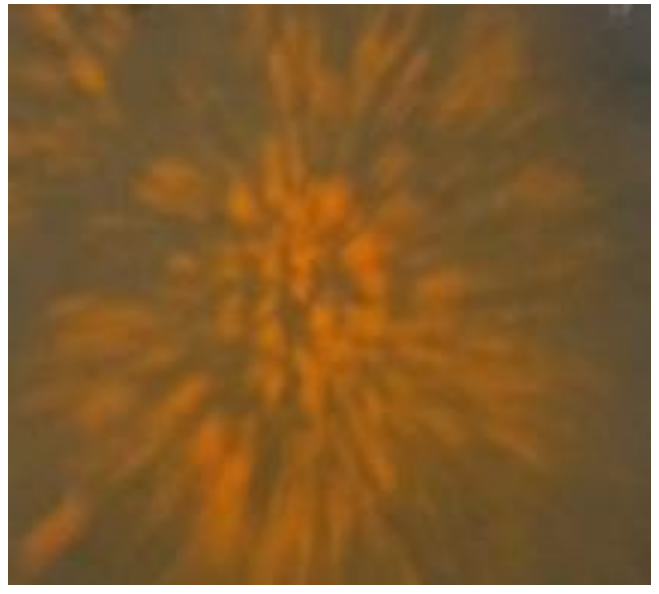

Figure 2. The spontaneous dispersion of turmeric powder of approximately 30 microns in size on water surface

The vertical component of the capillary force (FC) depends on the particle radius $\mathrm{R}$, the interfacial tension $\gamma$, the filling angle $\theta \mathrm{c}$ and the contact angle $\alpha$, and is given by $\mathrm{Fc}=-2 \pi \mathrm{R} \gamma \sin (\theta \mathrm{c}+\alpha)$ [20].

A DNS (Direct Numerical Simulation) approach was developed for particles trapped at fluid-fluid interface, for resolving the motion of a particle as well as the clusters of the particles and the rapidly changing dynamics of the particles, the modeling of interactions among floating particles [10].

The frequency of oscillation of particles about their floating equilibrium increased with decreasing particle size when they are adsorbed on both air-water and liquid-liquid interfaces [25].

In this paper, the variation of oscillation frequency of the particles when come into contact with air-liquid interface is shown when there is a change in the temperature of the liquid on which the dispersion of the particles take place.

\section{EXPERIMENTAL SETUP}

The experimental setup used in this study is shown in Figure 3. A petri dish is filled with the liquid to a height of $80 \%$ of the total height of the petri dish, approximately. For this study, Millipore water was used in order to ensure that contaminants were not present since their presence even in small amount on the air-water interface might change the interfacial tension and the contact angle of the liquid. Moreover, the particles and powders which were used in the experiment were dried in the oven to overcome any influence of the moisture over the contact angle and hence the position of the three-phase contact line on the particle's surface. The horizontal and vertical positions of particles were recorded using a digital video camera (Sony RX100).

The particles were released over the liquid surface approximately $1 \mathrm{~mm}$ in order to avoid any gain in the momentum due to the height from the surface. The motion of the particles was observed and measured by analyzing the videos that were recorded by the camera frame-by-frame with a calibrated digital ruler. The vertical and horizontal positions of particles were measured as a function of time by analyzing the video recordings. 


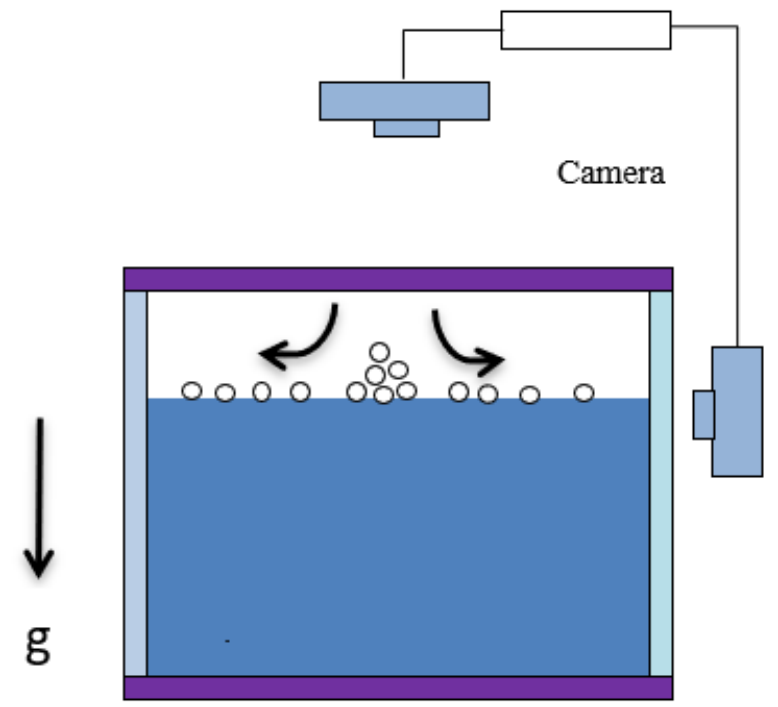

Figure 3. Schematic of the experimental setup used to study the dispersion of particles on a fluid-liquid interface.

\section{ADSORPTION OF PARTICLES ON A LIQUID SURFACE AND THE TEMPERATURE INFLUENCE OF LIQUID}

It has been already shown that while a particle is adsorbed on the surface of a liquid it oscillates about its equilibrium position, inducing a flow diametrically outward from the particle itself on the interface before the oscillations become zero. This induced flow moves other particles on the interface away. To investigate the influence of the liquid surface temperature on these oscillations, a plastic bead of size $3 \mathrm{~mm}$ was dropped from a height of 2 to $3 \mathrm{~mm}$ from the liquid surface on a container which was filled ( $90 \%$ of the volume) with water at $24^{\circ} \mathrm{C}$. The particle was dropped from such a close height gently so as to avoid any momentum gain due to the height. This was recorded vertically from the side of the experimental setup so as to view the oscillations of the particle clearly and closely. The particle was pulled by the capillary force when it came in contact with the liquid surface which led to oscillations of it about the equilibrium height vertically due to the reversal of the direction of vertical component of capillary force acting on the particle after it passed the equilibrium height, and the buoyant weight of the particle, and the viscous drag of the liquid. The balance of these forces led to the equilibrium position of the particle on the interface. The figure.4(a) shows the oscillation of the bead with different time intervals very clearly. The calculation of the frequency of oscillation was carried out by observing the time taken for the particle to complete one full cycle of oscillation from the moment it came in contact with the water surface until it completed one full oscillation vertically about the equilibrium height. The particle is considered to have completed one full cycle from the position it touches the airwater interface, until it reaches the maximum upward position after going down and coming up. The experiment was repeated filling the container with water at a temperature of $60^{\circ} \mathrm{C}$ and the frequency of oscillation was calculated. Figure 5 shows that the frequency of oscillation to be $7 \mathrm{~Hz}$ when the water was at $24^{\circ} \mathrm{C}$, whereas it is $3.5 \mathrm{~Hz}$ when the water was at $60^{\circ} \mathrm{C}$. The experiment was also performed with a particle of size $5.5 \mathrm{~mm}$ (Figure 4(b)). The frequency was calculated from the graph (Figure 6) to be $3.15 \mathrm{~Hz}$ when water was at $24^{\circ} \mathrm{C}$ and $2.6 \mathrm{~Hz}$ when water was at $60^{\circ} \mathrm{C}$. It clearly shows that the frequency of the oscillation changed with respect to the temperature of the liquid on which the adsorption occurred. These results show that the frequency of oscillation decreased with increase in the temperature of the water. This happens because of the decrease of surface tension of the air-water interface due to the influence of temperature which in turn decreases the capillary force which arises from the surface tension. 
Journal of Thermal Engineering, Technical Note, Vol. 5, No. 5, pp. 396-404, October, 2019

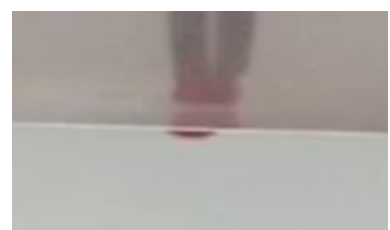

The particle is being adsorbed on the interface

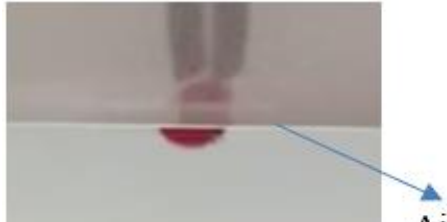

Air-water interface

After 0.033 seconds (going down)

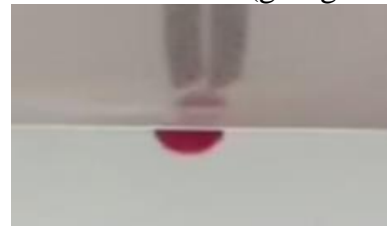

After 0.083 seconds (going further down)

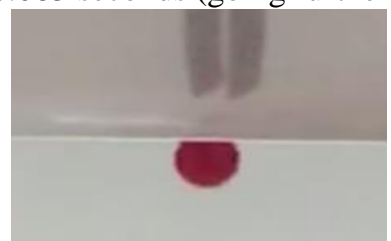

After 0.116 second (reaching maximum)

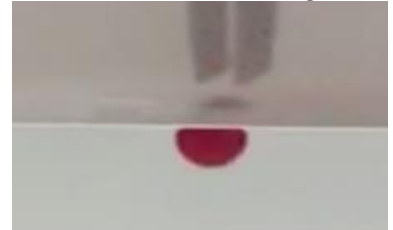

After 0.166 seconds (going up)

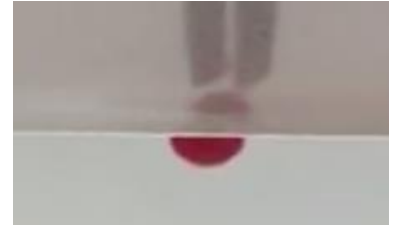

After 0.25 seconds (going up further)

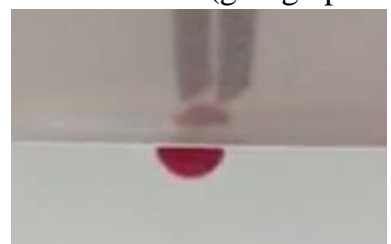

After 0.283 seconds (going down)

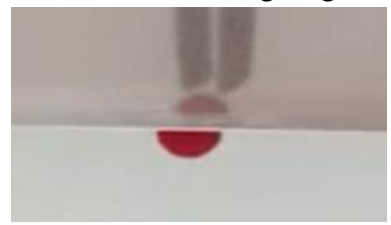

Final equilibrium position (a)

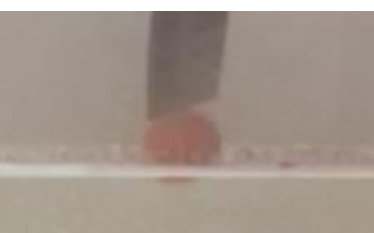

The particle is being adsorbed on the interface

After

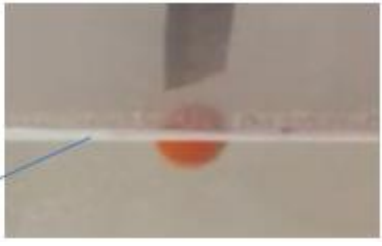

After 0.016 seconds (going down)

After 0.05 seconds (going further down)

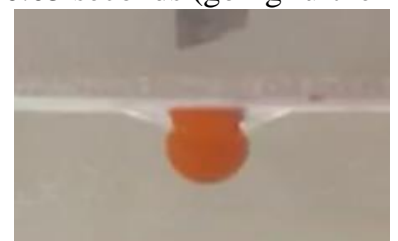

After 0.066 seconds (maximum position)

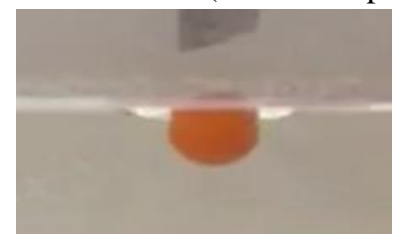

After 0.1 seconds (going up)

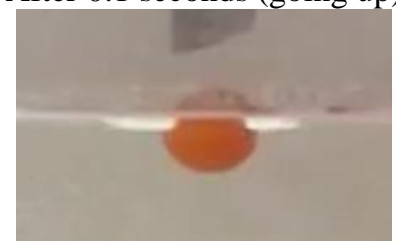

After 0.133 seconds (going up)

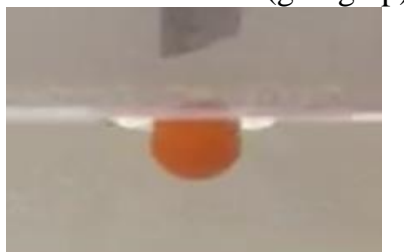

After 0.15 seconds (going down)

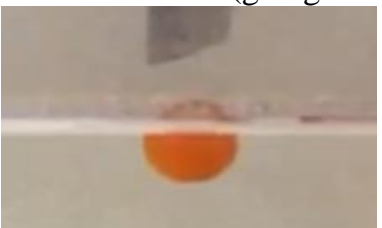

Final equilibrium position(b)

Figure 4. The adsorption of a $3 \mathrm{~mm}$ sized plastic bead on water surface at (a) $24^{\circ} \mathrm{C}$ and (b) $60^{\circ} \mathrm{C}$ 
Frequency of particle on air-liquid interface

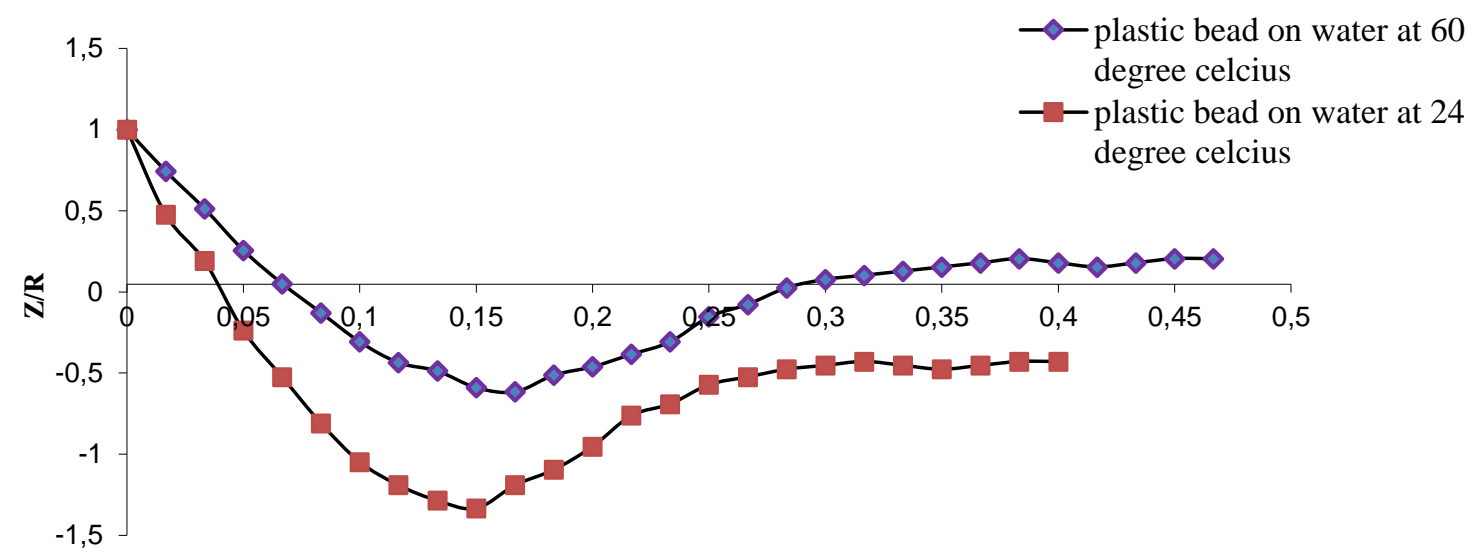

$\mathbf{t}(\mathbf{s})$

Figure 5. The variation of the frequency of oscillations of $3 \mathrm{~mm}$ particle with temperature on air-water interface

Frequency of particle on air-liquid interface

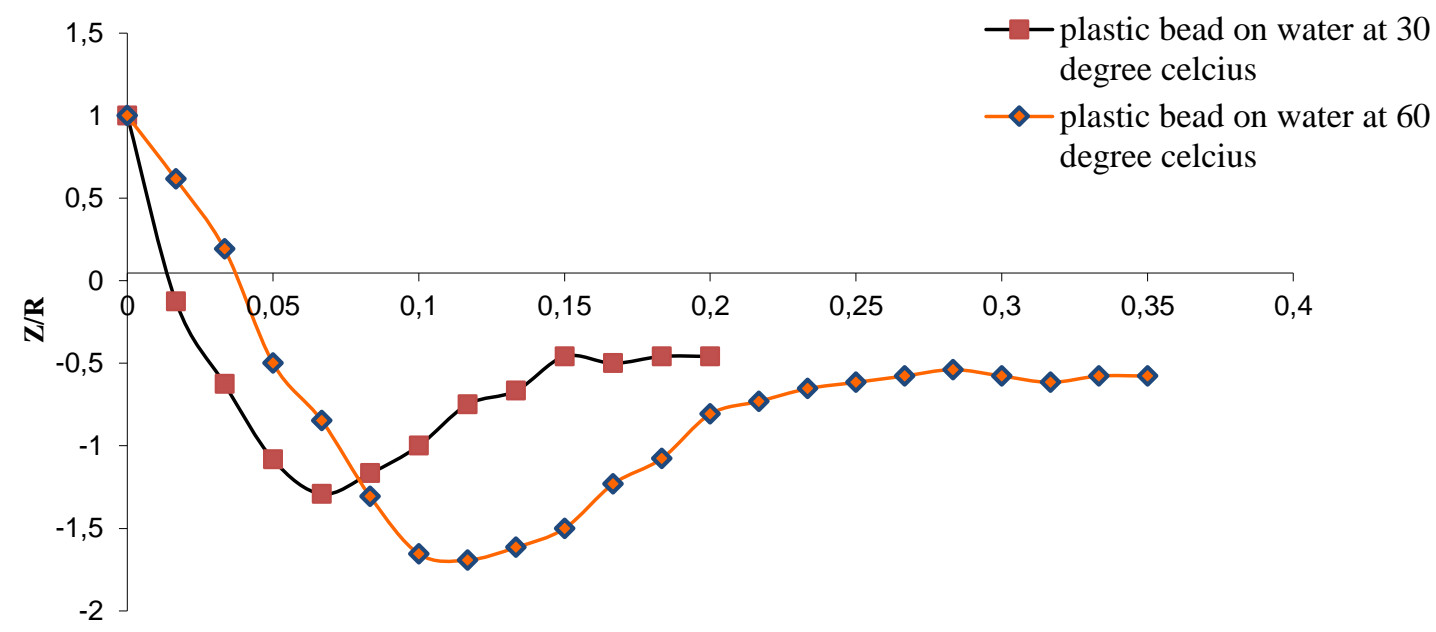

$\mathbf{t}(\mathbf{s})$

Figure 6. The variation of the frequency of oscillations of $5.5 \mathrm{~mm}$ particle with temperature on air-water interface

\section{ADSORPTION OF TWO PARTICLES}

Two plastic particles of the same size $(3 \mathrm{~mm})$ were dropped from a plastic straw, with a groove to hold the two particles together, on the water surface very close to the surface (less than $3 \mathrm{~mm}$ of height). The particles moved away from each other and came to rest after a while. This happened because of the vertical oscillation of the particles 
on the liquid surface about the equilibrium height which induced the flow on the liquid surface which pushed the particles away from each other. The particles stopped their motion after a while due to the viscous drag force which was acting on them. The figure.7 show the movement of the same sized particles with the same initial gap between them for the liquid's (water) temperatures of $24^{\circ} \mathrm{C}$ and $60^{\circ} \mathrm{C}$. The pictures show that there is no initial gap between the particles when they were touching the water surface and after 0.33 seconds the gap between the centers of the particle became $17.5 \mathrm{~mm}$ (for the particles that were at $24^{\circ} \mathrm{C}$ ), whereas the gap was $7.5 \mathrm{~mm}$ for the particles that were at $60^{\circ} \mathrm{C}$. The distance between the centers of the two particles became $35 \mathrm{~mm}$ when they dispersed on the water of temperature $24^{\circ} \mathrm{C}$, whereas the distance between the centers of the particles became $17.5 \mathrm{~mm}$ when the temperature of the water was $60^{\circ} \mathrm{C}$. This decrease in the gap between the particles that were dropped on the water at $60^{\circ} \mathrm{C}$ can be explained as followed. The initial velocity with which the particles were pulled into the interface was mainly by the vertical component of the capillary force which decreased when the temperature of the liquid was increased, and consequently the particles' frequency of oscillation about the interface also decreased, resulting in the dispersion rate of these two particles with a decrease in the gap between them.

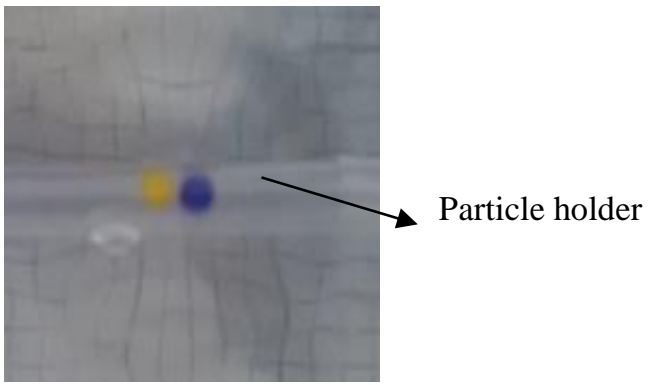

The particles touch the interface

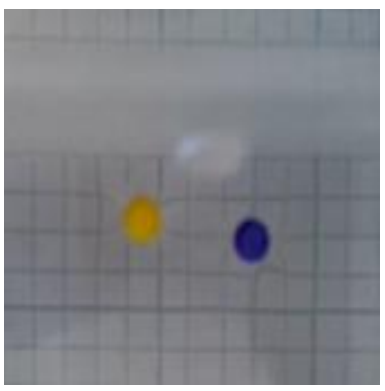

The dispersion after 0.33 seconds

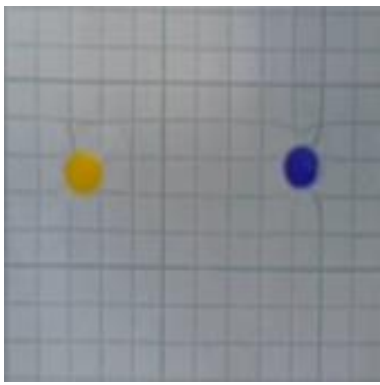

The final equilibrium position

(a)

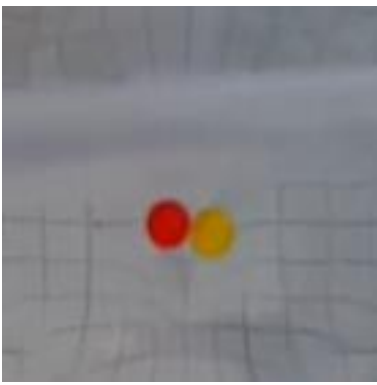

The particles touch the interface

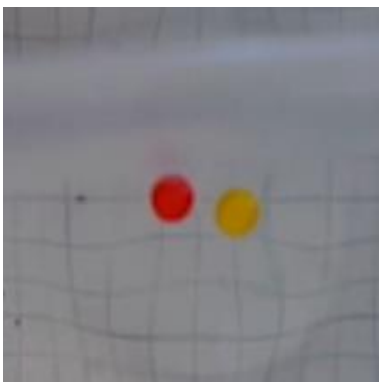

The dispersion after 0.33 seconds

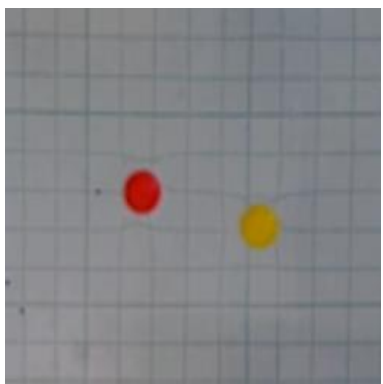

The final equilibrium position

(b)

Figure 7. The dispersion of two particles of size $3 \mathrm{~mm}$ on air-water interface with two different temperatures of the water (a) $24^{\circ} \mathrm{C}$ and (b) $60^{\circ} \mathrm{C}$ respectively 


\section{CONCLUSION}

Experiments reported in this paper show that when particles come in contact with air-liquid interface with different temperatures of the liquid, their spontaneous dispersion rate changes with the liquid's surface temperature. The experiments show that the dispersion rate decreases when the temperature of liquid surface on which the dispersion takes place increases. This occurs because for the same size and density of the particles, the magnitude of capillary force that the particles experience when they come in contact with the interface decreases due to the increased temperature of the liquid which in turn decreases the surface tension of the liquid and thereby the capillary force. The spontaneous dispersion which is the consequence of the flow that is induced on the liquid surface due to the vertical oscillations of the particles on the interface about their equilibrium height decreases due to this decrease in the capillary force. Though the drag force experienced by the particles might also vary due to the change in the temperature of the liquid surface, the influence by the change in the capillary force dominates the phenomenon which leads to the decrease in the dispersion rate.

\section{REFERENCES}

[1] Aubry, N., Singh, P., Janjua, M., \& Nudurupati, S. (2008). Assembly of defect-free particle monolayers with dynamically adjustable lattice spacing. Proceedings of the National Academy of Sciences, 105(10.1073), 3711-3714. [2] Venturi, M., Credi, A., \& Balzani, V. (2003). Molecular Devices and Machines—A Journey into the Nanoworld. Wiley-VCH, Weinheim.

[3] Bowden, N., Terfort, A., Carbeck, J., Whitesides, G. M. (1997). Self-assembly of mesoscale objects into ordered two-dimensional arrays. Science, 276(5310), 233-235.

[4] Cox, P. A., Knox, R. B. (1989). Two-dimensional pollination in hydrophilous plants: convergent evolution in the genera Halodule (Cymodoceaceae), Halophila (Hydrocharitaceae), Ruppia (Ruppiaceae), and Lepilaena (Zannichelliaceae). American journal of botany, 76(2), 164-175.

[5] Grzybowski, B. A., Bowden, N., Arias, F., Yang, H., Whitesides, G. M. (2001). Modeling of menisci and capillary forces from the millimeter to the micrometer size range. The Journal of Physical Chemistry B, 105(2), 404-412.

[6] Murray, C. B., Kagan, A. C., Bawendi, M. G. (2000). Synthesis and characterization of monodisperse nanocrystals and close-packed nanocrystal assemblies. Annual review of materials science, 30(1), 545-610.

[7] Nudurupati, S., Janjua, M., Singh, P., Aubry, N. (2010). Effect of parameters on redistribution and removal of particles from drop surfaces. Soft matter, 6(6), 1157-1169.

[8] Tang, Z., Zhang, Z., Wang, Y., Glotzer, S. C., Kotov, N. A. (2006). Self-assembly of CdTe nanocrystals into freefloating sheets. Science, 314(5797), 274-278.

[9] Wasielewski, M. R. (1992). Photoinduced electron transfer in supramolecular systems for artificial photosynthesis. Chemical Reviews, 92(3), 435-461.

[10] Singh, P., Joseph, D. D., Gurupatham, S. K., Dalal, B., Nudurupati, S. (2009). Spontaneous dispersion of particles on liquid surfaces. Proceedings of the National Academy of Sciences, 106(47), 19761-19764.

[11] Aveyard, R., Clint, J. H. (1996). Particle wettability and line tension. Journal of the Chemical Society, Faraday Transactions, 92(1), 85-89.

[12] Binks, B. P. (2002). Particles as surfactants—similarities and differences. Current opinion in colloid \& interface science, 7(1-2), 21-41.

[13] Chan, D. Y. C., Henry Jr, J. D., White, L. R. (1981). The interaction of colloidal particles collected at fluid interfaces. Journal of Colloid and Interface Science, 79(2), 410-418.

[14] Fortes, M. A. (1982). Attraction and repulsion of floating particles. Canadian Journal of Chemistry, 60(23), 28892895.

[15] Gifford, W. A., Scriven, L. E. (1971). On the attraction of floating particles. Chemical Engineering Science, 26(3), 287-297.

[16] Katoh, K., Fujita, H., Imazu, E. (1992). Motion of a particle floating on a liquid meniscus surface. Journal of fluids engineering, 114(3), 411-416.

[17] Kralchevsky, P. A., Paunov, V. N., Ivanov, I. B., Nagayama, K. (1992). Capillary meniscus interaction between colloidal particles attached to a liquid —-fluid interface. Journal of Colloid and Interface Science, 151(1), 79-94. 
[18] Lehle, H., Oettel, M. (2007). Importance of boundary conditions for fluctuation-induced forces between colloids at interfaces. Physical Review E, 75(1), 011602.

[19] Nicolson, M. M. (1949, April). The interaction between floating particles. In Mathematical Proceedings of the Cambridge Philosophical Society (Vol. 45, No. 2, pp. 288-295). Cambridge University Press.

[20] Singh, P., Joseph, D. D. (2005). Fluid dynamics of floating particles. Journal of Fluid Mechanics, 530, 31-80.

[21] Bresme, F., Oettel, M. (2007). Nanoparticles at fluid interfaces. Journal of Physics: Condensed Matter, 19(41), 413101.

[22] Kralchevsky, P. A., Denkov, N. D. (2001). Capillary forces and structuring in layers of colloid particles. Current opinion in colloid \& interface science, 6(4), 383-401.

[23] Paunov, V. N., Kralchevsky, P. A., Denkov, N. D., Nagayama, K. (1993). Lateral capillary forces between floating submillimeter particles. Journal of colloid and interface science, 157(1), 100-112.

[24] Stamou, D., Duschl, C., Johannsmann, D. (2000). Long-range attraction between colloidal spheres at the airwater interface: The consequence of an irregular meniscus. Physical Review E, 62(4), 5263-5272.

[25] Gurupatham, S., Dalal, B., Hossain, M. S., Fischer, I. S., Singh, P., Joseph, D. D. (2011). Particles dispersion on fluid-liquid interfaces. Particuology, 9(1), 1-13. 